\title{
一种复杂曲面类增材制造零件分层截面生成算法
}

\author{
韩兴国 $^{1,2}$ 宋小辉 $^{2}$ 殷 鸣 ${ }^{1}$ 殷国富 ${ }^{1}$ \\ (1. 四川大学制造科学与工程学院 成都 610065; \\ 2. 桂林航天工业学院机械工程学院 桂林 541004)
}

\begin{abstract}
摘要: 针对传统增材制造分层方法对复杂曲面类零件 STL 模型分层易造成部分层面轮廓线失真的问题, 提出一种基于 NURBS 曲线的复杂曲面类零件分层截面轮廓生成算法。针对 STL 模型数据量大和分层速度慢的特点, 采用了分组排序的求交算法进 行分层, 生成截面轮廓点云数据。以切平面与 STL 模型三角面片的交点作为 NURBS 曲线的型值点, 设定型值点的权值, 利 用矩阵形式和切失边界条件确定了 NURBS 曲线的权因子, 求解 NURBS 曲线的控制顶点, 采用矩阵形式建立了各切片层的 截面轮廓所对应的 NURBS 曲线方程, 绘制了基于 NURBS 曲线的各层层面轮廓。采用基于 NURBS 曲线的复杂曲面类零件 分层截面生成算法对燃气轮机中压缸动叶片和 Ganesha 模型进行了分层试验仿真和误差分析。进行了燃气轮机中压缸动叶片 的打印试验, 通过测量表明: 采用本算法打印叶片的轮廓度偏差值符合要求, 相比传统算法打印的叶片精度更高, 从而验证 了所提出的基于 NURBS 曲线的复杂曲面类零件分层截面生成算法的可行性和准确性。
\end{abstract}

关键词：增材制造；复杂曲面；分层算法；分组排序；非均匀有理 B 样条

中图分类号: TG156

\section{A Layered Section Contour Generation Algorithm for Additive Manufacturing Parts with Complex Surfaces}

\author{
HAN Xingguo $^{1,2}$ SONG Xiaohui $^{2}$ YIN Ming ${ }^{1} \quad$ YIN Guofu $^{1}$ \\ (1. School of Manufacturing Science and Engineering, Sichuan University, Chengdu 610065; \\ 2. Department of Mechanical Engineering, Guilin University of Aerospace Technology, Guilin 541004)
}

\begin{abstract}
Aiming at the problem of the traditional additive manufacturing slicing algorithm easily leading to the distortion of the contour lines of the STL models of complex surface parts, a layered section contour generation algorithm for complex surface parts based on the NURBS curve is proposed. Aiming at the characteristics of large quantity of STL model data and slow layering speed, an intersection algorithm based on grouping and ranking is used to layer models, which could generate the point cloud data of the cross-sectional profile. The intersection points of the tangent plane and the STL triangle facet are used as the data points of the NURBS curve, and the weight of the data points is set. The weight factor of the NURBS curve is determined by the matrix form and the cut loss boundary conditions, and the control vertices of the NURBS curve is solved. The NURBS curve equation corresponding to the contour lines of each slicing layer are established in a matrix form, and the layer contours based on the NURBS curve are drawn. The layered section contour generation algorithm for complex surface parts based on NURBS curves is adopted to perform layering simulation and error analysis on the gas turbine medium-pressure cylinder moving blade and Ganesha model. Finally, a printing experiment of the gas turbine medium-pressure cylinder moving blade is performed. The test results show that profile deviation value of the blade printed by the algorithm of this study met the requirements and its accuracy is higher compared with the blade printed by traditional algorithm. It can be seen that layered section contour generation algorithm for complex surface parts based on the NURBS curve proposed here is feasible and accurate.
\end{abstract}

Key words: additive manufacturing; complex surface; slicing algorithm; grouping and ranking; non-uniform rational b-spline

* 国家工信部智能制造新模式应用项目(2017bwqt-005-gxb)、国家自然 科学基金(51705094)、四川省智能制造专项课题(2016CD00478)、广 西高校中青年教师基础能力提升(KY2016YB535)和广西自然科学基金 (2018UA160218)资助项目。20180922 收到初稿, 20190304 收到修改稿 


\section{0 前言}

增材制造(又称 3D 打印)是以三维实体模型为 基础, 将打印材料层层堆积、固化、成型从而加工 出实体零件的一种制造技术 ${ }^{[1]}$, 其对传统的加工工 艺、生产模式、产品设计、生产线等产生了深远的 影响 ${ }^{[2]}$ 。

对增材制造实体模型沿分层方向进行离散化处 理的方法, 称为增材制造的分层算法 ${ }^{[3]}$ 。分层处理 采用平行于 $X Y$ 平面的切平面切割三维实体模型并 获得各层的层面轮廓位置信息, 将三维加工转化为 在二维平面内的逐层加工, 简化了加工的工艺 ${ }^{[4]}$ 。 通过切片分层获取实体模型在各切平面的层面轮廓 位置信息是实现增材制造的前提, 分层所得到的层 面轮廓位置信息的精度和质量最终影响着增材制造 产品的精度和质量。

增材制造分层算法主要包括基于 STL 模型的分 层算法 ${ }^{[5]}$ 和基于 $\mathrm{CAD}$ 模型的直接分层算法 ${ }^{[6-8]}$, 而 前者是目前主流的分层算法。

针对 STL 模型的分层算法, 一些学者建立了三 角面片的拓扑关系以便于分层。ROCK 等 ${ }^{[9]}$ 通过建 立离散的三角面片的拓扑信息, 实现了 STL 模型的 高效分层, 每个分层平面与各三角面片相交生成两 个交点, 将这些交点首尾相连形成一个封闭的路廓 线, 这种分层方法, 拓扑信息的建立至关重要, 直 接影响到查找三角面片的效率。张必成等 ${ }^{[10]}$ 以 STL 模型为研究对象, 提出了一种快速构建拓扑信息的 新算法, 提高了网格简化的效率。谢存禧等 ${ }^{[1]}$ 提出 了建立三角面片的邻接图表以提高搜索效率和分层 速度。

一些学者提出了基于三角面片位置信息的分层 算法。TATA 等 ${ }^{[12]}$ 提出先将三角面片按照顶点的 $z$ 值进行分类和排序, 将三角面片有序化, 在分层过 程中通过分层平面的高度和某面片 $z$ 值大小比较, 对某些三角面片的相交关系进行判定, 最终生成轮 廓线。

在自适应分层方面, ZHANG 等 ${ }^{[13]}$ 提出了一种 基于 STL 格式文件的自适应分层算法, 能自动识别 各层的内外封闭曲线, 提高分层效率。SABOURIN 等 ${ }^{[14-15]}$ 和 CORMIER 等 ${ }^{[16]}$ 提出了一种分层厚度根据 打印零件的曲面曲率情况自动调节的分层算法, 有 效地改善了零件的成型质量。PANDEY 等 ${ }^{[17]}$ 提出了 一种熔融沉积式增材制造分层算法, 根据每层边缘 轮廓的实时变化, 自动调节分层厚度, 获取较好的 表面质量和尺寸精度。
在提高分层面轮廓线的精度方面，刘斌等 ${ }^{[18]}$ 介 绍了一种增材制造实时切片算法, 对切片轮廓线误 差进行理论分析, 提出了一种提高切片轮廓线精度 的解决办法。

采用上述分层算法对一般的规则零件而言，所 生成的截面轮廓与该层的实际轮廓相比, 误差不大, 但对于一些复杂曲面类零件, 曲面部分应比平面部 分的转换精度要求高, 如果采用上述的基于 STL 模 型分层方法对复杂曲面类零件易造成分层面的轮廓 线失真，产生一定的精度损失。基于此，本文提出 了一种基于 NURBS 曲线的复杂曲面类零件分层截 面生成算法。

\section{STL 模型分层算法的基本原理}

\section{1 分层算法流程}

基于 STL 模型的分层算法首先要确定分层方向 和分层厚度 $\Delta Z$, 进而沿分层方向(间隔为 $\Delta Z$ ) 逐一求 取切平面与三维模型的交线, 将交线依次连接形成 封闭曲线, 即切片层的层面轮廓。分层算法控制流 程如图 1 所示。

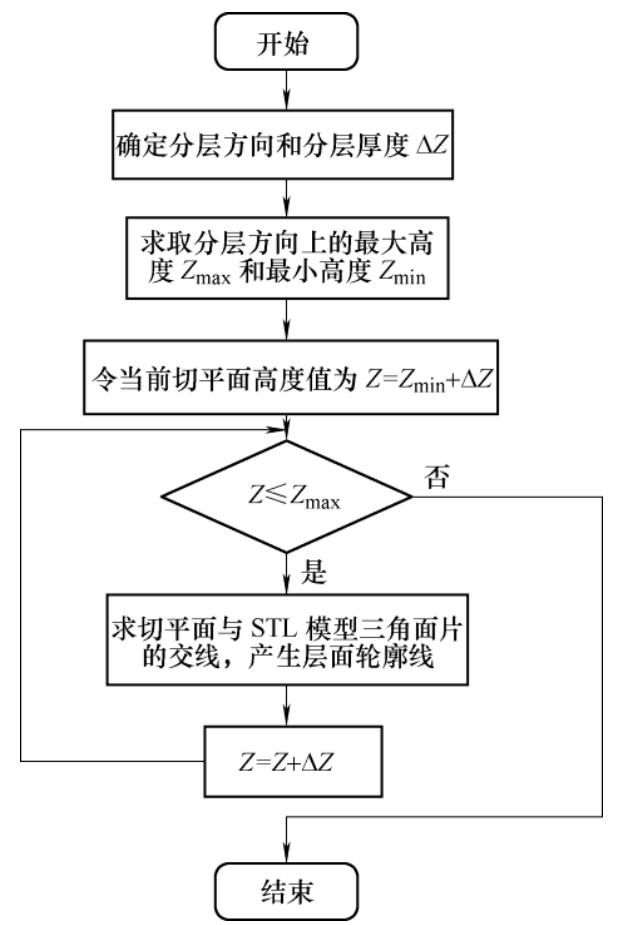

图 1 STL 模型分层算法流程

由图 1 可以看出, 影响 STL 模型分层算法速 度的因素有: (1) 判断切平面和三角面片的空间位 置关系; (2) 对切平面和三角面片进行求交运算; (3) 生成各截面的封闭轮廓线。所以, 提高 STL 模型分层算法的效率需要综合考虑这三个因素的 影响。 


\section{2 分组排序}

根据 STL 模型分层算法的流程, 本文提出了基 于分组排序的求交算法。该算法的基本思想是：根 据 STL 模型的几何连续性对各三角面片进行分组和 排序，构建分层关系矩阵，进而对三角面片进行对 边求交，最终生成各截面的封闭轮廓线。

假设切平面的数量为 $n$, 那么可将三角面片分 为 $n$ 组, 查找三角面片上 3 个顶点在分层方向上的 最小值, 确定该三角面片最初出现的层号。把与同 一切平面相交的所有三角面片归为一组, 遍历 STL 模型的所有三角面片, 构建以分层号为索引的三角 面片集合，这就是分层关系矩阵。

图 2 为分层关系矩阵的示意图, $F_{i}(i=1,2, \cdots$, $n$ )代表与层号为 $i$ 的切平面相交的三角面片集合, 则 $F_{i}=\left(f_{i 1}, f_{\mathrm{i} 2}, \cdots, f_{i m}\right), f_{i m}$ 为与第 $i$ 个切平面相交的第 $m$ 个三角面片, 与当前切平面相交的的所有三角面 片的集合构成了活性三角面片表。

\begin{tabular}{|c|c|c|c|c|c|}
\hline$i=n$ \\
\hdashline$\vdots$ \\
\hline$i=3$ \\
\hline$i=2$ \\
\hline$i=1$ \\
\hline$f_{n 1}$ & $f_{n 2}$ & $f_{n 3}$ & $\cdots$ & $f_{n m_{n}}$ \\
\hline$f_{21}$ & $f_{22}$ & $f_{23}$ & $\cdots$ & $f_{2 m_{2}}$ \\
\hline$f_{11}$ & $f_{12}$ & $f_{13}$ & $\cdots$ & $f_{1 m_{1}}$ \\
\hline
\end{tabular}

图 2 分层关系矩阵示意图

通过建立分层关系矩阵和活性三角面片表, 判 断切平面和三角面片在空间中位置关系的次数大大 减少, 有助于提高 STL 模型的分层速度。

\section{3 对边求交}

通过求交运算可以获取层面轮廓, 通过求取分 层关系矩阵中各三角面片的边与切平面的交点, 将 处于同一层的所有交点依次排列, 即可构成各层面 轮廓的点云数据。图 3 为切平面与三角面片交线形 成的示意图。

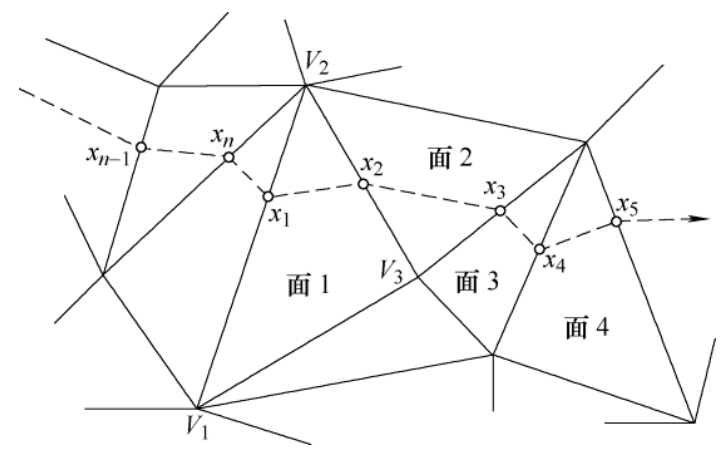

图 3 切平面与三角面片的交线
为了提高分层处理速度和减少数据处理环节, 在求出切平面与第一个三角面片的第一条边的交点 后(图 3 中的点 $X_{1}$ ), 应根据该面片的外法向量判断 出第二个交点所在的方向。判断出 $X_{2}$ 的方向后, 即 可判断出第二条需要求交的边, 求出切平面和第二 条边的交点。按照同样的方法判断出下一条需要求 交的边, 并求出交点, 依次追踪, 直到回到第一个 相交点 $X_{1}$ 。当求出某个三角面片各边与切平面的交 点之后, 必须将该三角面片从分层关系矩阵中去除, 避免重复搜索，提高分层速度。

采用分组排序的分层算法对 STL 模型进行分 层, 依次求取切平面与三角面片的交点, 用直线依 次连接各交点所形成的轮廓即为该层的截面轮廓, 这能够提高分层的效率，但不能提高分层精度。因 此, 本文采用 NURBS 曲线对各层的交点进行逼近 拟合，获取更加准确的分层曲面，有助于提升分层 精度和分层质量。

\section{2 基于 NURBS 曲线的复杂曲面类 零件分层截面生成算法}

采用基于 STL 模型的分层算法, 某一切平面与 零件模型相交，求取该切平面与所有三角面片的交 点, 用直线依次连接各交点所形成的轮廓即为该层 的截面轮廓, 对复杂曲面类零件而言, 采用直线相 连的方法构建的截面轮廓存在失真问题。基于此, 本文提出了一种基于 NURBS 曲线的复杂曲面类零 件分层截面生成算法。

\subsection{NURBS 曲线基本概念}

NURBS 曲线又称之为非均匀有理 B 样条(Non uniform rational B-spline, NURBS)曲线, 德布尔首先 提出了 B 样条的标准算法, 后经多位学者改进和推 动, NURBS 曲线应用更加广泛 ${ }^{[19]}$ 。

一条 $k$ 次 NURBS 曲线可以表示为

$$
p(u)=\frac{\sum_{i=0}^{n} \omega_{i} d_{i} N_{i, k}(u)}{\sum_{i=0}^{n} \omega_{i} N_{i, k}(u)} 0 \leqslant u \leqslant 1
$$

式中, $k$ 为 NURBS 曲线的阶次, $\omega_{i}(i=0,1, \cdots, n)$ 称 为权因子, $d_{i}(i=0,1, \cdots, n)$ 称为控制顶点, $i$ 为样条基 函数的编号, $n$ 为样条基函数样条数量, $u$ 为 NURBS 曲线的参变量, $u \in\left[u_{i}, u_{i+1}\right], N_{i, k}(u)$ 是由节点矢量 $U=\left[u_{0}, u_{1}, \cdots, u_{n+k+1}\right]$ 按德布尔-考克思递推公式 ${ }^{[19]}$ 所 确定的 $k$ 次规范 $\mathrm{B}$ 样条基函数，如下所示 


$$
\left\{\begin{array}{l}
N_{i, 0}(u)= \begin{cases}1 & u_{i} \leqslant u \leqslant u_{i+1} \\
0 & \text { 其他 }\end{cases} \\
N_{i, k}(u)=\frac{u-u_{i}}{u_{i+k}-u_{i}} N_{i, k}(u)+\frac{u_{i+k+1}-u}{u_{i+k+1}-u_{i+1}} N_{i+1, k-1}(u)(2) \\
\text { 确定 } \frac{0}{0}=0
\end{array}\right.
$$

\subsection{NURBS 曲线的矩阵表示形式}

已知 NURBS 曲线的型值点, 当进行反求、拟 合时, 由于数据量庞大, 如果采用有理多项式(式(1)) 对 NURBS 曲线求值、求导, 计算量较大。本文采 用 NURBS 曲线的矩阵形式，使用更为方便。

$k$ 次曲线上的参数 $u \in\left[u_{i}, u_{i+1}\right]$ 的一点 $p(u)$ 至多与 $k+1$ 个顶点 $d j(j=i-k, i-k+1, \cdots, i)$ 有关, 而与其他顶点

$$
\begin{gathered}
p_{i}(u)=\frac{\sum_{j=i-3}^{i} \omega_{j} d_{j} N_{j, 3}(u)}{\sum_{j=i-3}^{i} \omega_{j} N_{j, 3}(u)}=\frac{\omega_{i-3} d_{i-3} N_{i-3,3}(u)+\omega_{i-2} d_{i-2} N_{i-2,3}(u)+\omega_{i-1} d_{i-1} N_{i-1,3}(u)+\omega_{i} d_{i} N_{i, 3}(u)}{\omega_{i-3} N_{i-3,3}(u)+\omega_{i-2} N_{i-2,3}(u)+\omega_{i-1} N_{i-1,3}(u)+\omega_{i} N_{i, 3}(u)} \\
p_{i}(u)=\frac{\left[N_{i-3,3}(u) N_{i-2,3}(u) N_{i-1,3}(u) N_{i, 3}(u)\right]\left[\begin{array}{c}
\omega_{i-3} d_{i-3} \\
\omega_{i-2} d_{i-2} \\
\omega_{i-1} d_{i-1} \\
\omega_{i} d_{i}
\end{array}\right]}{\left[N_{i-3,3}(u) N_{i-2,3}(u) N_{i-1,3}(u) N_{i, 3}(u)\right]\left[\begin{array}{c}
\omega_{i-3} \\
\omega_{i-2} \\
\omega_{i-1} \\
\omega_{i}
\end{array}\right]}
\end{gathered}
$$

为简化计算, 引入算子 $\Delta$, 并规定

$$
\begin{gathered}
4=u_{i+1}-u_{i} \quad i=0,1, \cdots, n+k \\
\Delta_{i}^{2}=\Delta_{i}+\Delta_{i+1}=u_{i+2}-u_{i} \quad i=0,1, \cdots, n+k-1 \\
\Delta_{i}^{3}=\Delta_{i}+\Delta_{i+1}+\Delta_{l+2}=u_{i+3}-u_{i} i=0,1, \cdots, n+k-2
\end{gathered}
$$

同理可得

$$
4_{i}^{k}=4_{i}+4_{i+1}+\cdots+\Delta_{+k-1}=u_{i+k}-u_{i}
$$

规定: $4_{0}=0$ 。

由式 (2) 的德布尔-考辛斯递推公式求出 $N_{i-3,3}(u), N_{i-2,3}(u)$ 和 $N_{i-1,3}(u)$ 和 $N_{i, 3}(u)$ 的表达式, 代

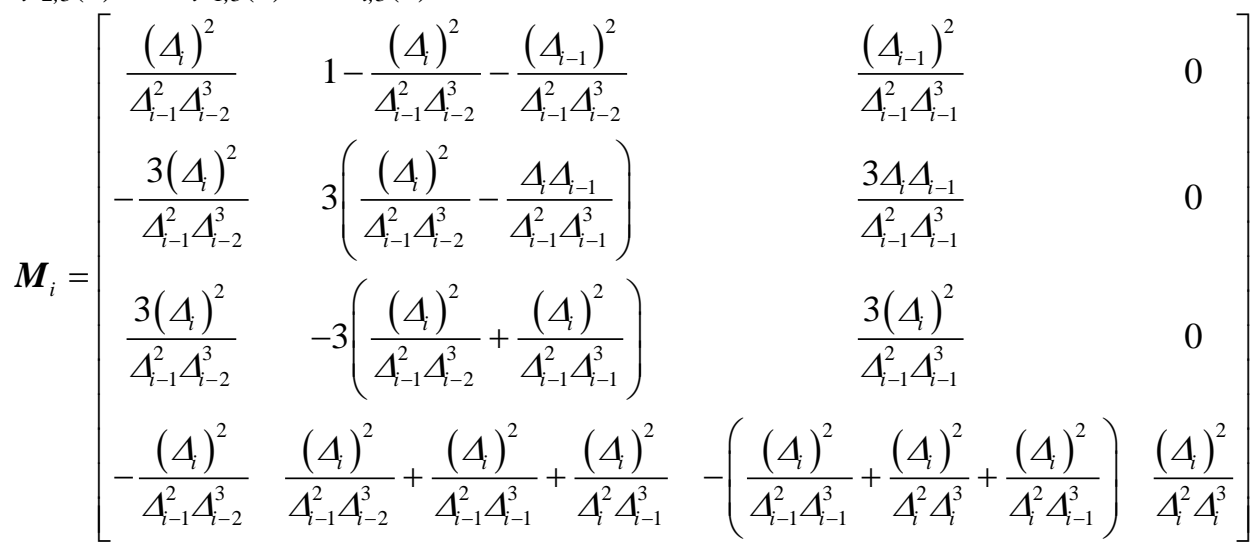

入式(5)可得三次 NURBS 曲线的矩阵形式为

$$
\begin{gathered}
p_{i}(t)=\frac{\boldsymbol{T}_{3} \boldsymbol{M}_{i} \boldsymbol{D}_{3}}{\boldsymbol{T}_{3} \boldsymbol{M}_{i} \boldsymbol{W}_{3}} \\
t=\frac{u-u_{i}}{u_{i+1}-u_{i}} \quad 0 \leqslant u \leqslant 1 \\
\boldsymbol{D}_{3}=\left[\begin{array}{llll}
\omega_{i-3} d_{i-3} & \omega_{i-2} d_{i-2} & \omega_{i-1} d_{i-1} & \omega_{i} d_{i}
\end{array}\right]^{\mathrm{T}} \\
\boldsymbol{W}_{3}=\left[\begin{array}{llll}
\omega_{i-3} & \omega_{i-2} & \omega_{i-1} & \omega_{i}
\end{array}\right]^{\mathrm{T}}
\end{gathered}
$$

S 曲线的 参变量 $u$, 从理论上讲, NURBS 曲线的阶次越高, 增加，影响分层效率，本文综合考虑拟合精度和拟 合的运算量, 选择 NURBS 曲线的阶次为 3 。根据 式(2)和式(3), 当 $k=3$ 时, 得到第 $i$ 段三次 NURBS 曲线, 如式(4)所示。将式(4)转换为矩阵形式, 如式 (5)所示
式中, $i=3,4, \cdots, n+1, \boldsymbol{T}_{3}=\left[\begin{array}{llll}1 & t & t^{2} & t^{3}\end{array}\right]$ 。

引入算子 $\Delta, M_{i}$ 可以转化如式(14)所示 


\subsection{NURBS 曲线的反算}

已知控制点和节点矢量, 求 NURBS 曲线上的 点和各阶导失称为正算, 已知 NURBS 曲线上的型 值点(数据点), 求 NURBS 曲线的节点矢量和控制顶 点称为反算问题。

假设已知型值点 $P_{i}(i=0,1, \cdots, m)$, 若要求解控制 顶点 $d_{i}(i=0,1, \cdots, n)$, 其中 $n=m+k-1$, 必须先求解出 NURBS 曲线的节点矢量, 本文采用向心参数法 ${ }^{[20]}$ 求解节点矢量。令

$$
\begin{gathered}
D=\sum_{h=1}^{m} \sqrt{\left|P_{h}-P_{h-1}\right|} \\
\text { 令 } \bar{u}_{0}=0, \quad \bar{u}_{n}=1 \\
\bar{u}_{h}=\bar{u}_{h-1}+\frac{\sum_{h=1}^{m} \sqrt{\left|P_{h}-P_{h-1}\right|}}{D} \quad h=1,2, \cdots, n-1
\end{gathered}
$$

NURBS 曲线的节点矢量为 $U=\left[u_{0}, u_{1}, \cdots, u_{n+k+1}\right]$, $k$ 为 NURBS 曲线的阶次, 根据节点矢量的定义, 可 得 $u_{0}=u_{1}=\cdots=u_{k}=0, u_{n+1}=u_{n+2}=\cdots=u_{n+k+1}=1$, 则

$$
u_{j+k}=\frac{1}{k} \sum_{i=j}^{j+k-1} \overline{u_{i}} \quad j=1,2, \cdots, n-k+1
$$

根据式(17)可以求解出 $u_{k+1}, u_{k+2}, \cdots, u_{n}$ 的值, 根 据式(10), 可得

$$
p_{i}(t)=\frac{\left[\begin{array}{cccc}
1 & t & t^{2} & t^{3}
\end{array}\right] M_{i}\left[\begin{array}{llll}
\omega_{i-3} d_{i-3} & \omega_{i-2} d_{i-2} & \omega_{i-1} d_{i-1} & \omega_{i} d_{i}
\end{array}\right]^{\mathrm{T}}}{\left[\begin{array}{lllll}
1 & t & t^{2} & t^{3}
\end{array}\right] M_{i}\left[\begin{array}{lllll}
\omega_{i-3} & \omega_{i-2} & \omega_{i-1} & \omega_{i}
\end{array}\right]^{\mathrm{T}}}
$$

把三次 NURBS 曲线的首末控制点作为首末插 值点, 根据插值要求可得

$$
\left\{\begin{array}{l}
p_{3}(0)=P_{0} \\
p_{i}(1)=p_{i+1}(0)=P_{i-2} \\
p_{n}(1)=P_{m}
\end{array}\right.
$$

由式(11)可知, 当 $u=u_{i}$ 时, $t=0$, 根据式(18)可 得式(20)。

根据式(14)和式(20)可得式(21)。

$$
\begin{aligned}
& P_{i-3}=p_{i}(0)=\frac{\left[\begin{array}{cccc}
1 & 0 & 0 & 0
\end{array}\right] M_{i}\left[\begin{array}{llll}
\omega_{i-3} d_{i-3} & \omega_{i-2} d_{i-2} & \omega_{i-1} d_{i-1} & \omega_{i} d_{i}
\end{array}\right]^{\mathrm{T}}}{\left[\begin{array}{lllll}
1 & 0 & 0 & 0
\end{array}\right] M_{i}\left[\begin{array}{llll}
\omega_{i-3} & \omega_{i-2} & \omega_{i-1} & \omega_{i}
\end{array}\right]^{\mathrm{T}}} \\
& P_{i-3}=p_{i}(0)=\frac{\frac{\left(\Delta_{i}\right)^{2}}{\Delta_{i-1}^{2} \Delta_{i-2}^{3}} \omega_{i-3} d_{i-3}+\left(1-\frac{(4)^{2}}{\Delta_{i-1}^{2} \Delta_{i-2}^{3}}-\frac{\left(\Delta_{i-1}\right)^{2}}{\Delta_{i-1}^{2} \Delta_{i-2}^{3}}\right) \omega_{i-2} d_{i-2}+\frac{\left(\Delta_{i-1}\right)^{2}}{\Delta_{i-1}^{2} \Delta_{i-1}^{3}} \omega_{i-1} d_{i-1}}{\frac{\left(\Delta_{i}\right)^{2}}{\Delta_{i-1}^{2} \Delta_{i-2}^{3}} \omega_{i-3}+\left(1-\frac{\left(\Delta_{i}\right)^{2}}{\Delta_{i-1}^{2} \Delta_{i-2}^{3}}-\frac{\left(\Delta_{i-1}\right)^{2}}{\Delta_{i-1}^{2} \Delta_{i-2}^{3}}\right) \omega_{i-2}+\frac{\left(\Delta_{i-1}\right)^{2}}{\Delta_{i-1}^{2} \Delta_{i-1}^{3}} \omega_{i-1}}
\end{aligned}
$$

令

$$
\begin{gathered}
a_{i-2}=\frac{\left(\Delta_{1}\right)^{2}}{\Delta_{i-1}^{2} \Delta_{i-3}^{3}} \omega_{i-3} \\
b_{i-2}=\left(1-\frac{\left(\Delta_{i}\right)^{2}}{\Delta_{i-1}^{2} \Delta_{i-2}^{3}}-\frac{\left(\Delta_{i}\right)^{2}}{\Delta_{i-1}^{2} \Delta_{i-2}^{3}}\right) \\
c_{i-2}=\frac{\left(\Delta_{i-1}\right)^{2}}{4_{i-1}^{2} \Delta_{i-1}^{3}} \omega_{i-1}
\end{gathered}
$$

可得

$$
a_{i-2} d_{i-3}+b_{i-2} d_{i-2}+c_{i-2} d_{i-1}=\left(a_{i-2}+b_{i-2}+c_{i-2}\right) P_{i-3}
$$

式中, $i=3,4, \cdots, n+1$, 共包含 $m+1=n-1$ 个方程, 共有 $n+1$ 个未知量, 需要增加两个端点条件才能对 方程组进行求解。

式(22)可用矩阵形式表示为

$$
\left(\begin{array}{cccccc}
b_{0} & c_{0} & & & & \\
a_{1} & b_{1} & c_{1} & & & \\
& a_{2} & b_{2} & c_{2} & & \\
& & \ddots & \ddots & \ddots & \\
& & & a_{n-1} & b_{n-1} & c_{n-1} \\
& & & & a_{n} & b_{n}
\end{array}\right)\left(\begin{array}{c}
d_{0} \\
d_{1} \\
d_{2} \\
\vdots \\
d_{n-1} \\
d_{n}
\end{array}\right)=\left(\begin{array}{c}
Q_{0} \\
Q_{1} \\
Q_{2} \\
\vdots \\
Q_{n-1} \\
Q_{n}
\end{array}\right)
$$

$$
Q_{i-2}=\left(a_{i-2}+b_{i-2}+c_{i-2}\right) P_{i-3} \quad i=3,4, \cdots, n+1
$$

式(23)系数矩阵中除第一行和第 $n+1$ 行的系数 $b_{0} 、 c_{0} 、 a_{n}$ 和 $b_{n}$ 外，其他系数可以通过式(21)和式(22) 求解， $b_{0} 、 c_{0} 、 a_{n}$ 和 $b_{n}$ 的求解需要通过边界条件补 充两个辅助方程进行求解，本文采用切失条件作为 边界条件进行求解。首末端附加方程如式(24)所示, 其中 $c_{0}^{\prime}$ 和 $c_{m}^{\prime}$ 为首末型值点 $c_{0}$ 和 $c_{m}$ 处的切矢, $k$ 为 NURBS 曲线的次数, $k$ 取 $3, m$ 为型值点的个数, 由切矢边界条件 ${ }^{[21-22]}$ 附加的方程为

$$
\left\{\begin{array}{l}
d_{1}-d_{0}=\frac{\omega_{0} \Delta_{3}}{3 \omega_{1}} c_{0} \\
d_{n}-d_{n-1}=\frac{\omega_{n+1} \Delta_{n}}{3 \omega_{n}} c_{m}
\end{array} .\right.
$$

式中, $c_{0}{ }^{\prime}=Q_{0}, c_{m}{ }^{\prime}=c_{n-2}{ }^{\prime}=Q_{n}$ 。

式(24)可以转化为

$$
\left\{\begin{array}{l}
-\frac{3 \omega_{1}}{\omega_{0} \Delta_{3}} d_{0}+\frac{3 \omega_{1}}{\omega_{0} \Delta_{3}} d_{1}=Q_{0} \\
-\frac{3 \omega_{n}}{\omega_{n+1} \Delta_{n+1}} d_{n-1}+\frac{3 \omega_{n}}{\omega_{n+1} \Delta_{n+1}} d_{n}=Q_{n}
\end{array}\right.
$$




$$
\left\{\begin{array}{l}
b_{0}=-\frac{3 \omega_{1}}{\omega_{0} \Delta_{3}} \\
c_{0}=\frac{3 \omega_{1}}{\omega_{0} \Delta_{3}} \\
a_{n}=-\frac{3 \omega_{n}}{\omega_{n+1} \Delta_{n+1}} \\
b_{n}=\frac{3 \omega_{n}}{\omega_{n+1} \Delta_{n+1}}
\end{array}\right.
$$

根据式(23)可得

$$
\left[\begin{array}{c}
d_{0} \\
d_{1} \\
d_{2} \\
\vdots \\
d_{n-1} \\
d_{n}
\end{array}\right]=\left[\begin{array}{cccccc}
b_{0} & c_{0} & & & & \\
a_{1} & b_{1} & c_{1} & & & \\
& a_{2} & b_{2} & c_{2} & & \\
& & \ddots & \ddots & \ddots & \\
& & & a_{n-1} & b_{n-1} & c_{n-1} \\
& & & & a_{n} & b_{n}
\end{array}\right]^{-1}\left[\begin{array}{c}
Q_{0} \\
Q_{1} \\
Q_{2} \\
\vdots \\
Q_{n-1} \\
Q_{n}
\end{array}\right]
$$

根据式(27) 可以求出 NURBS 的控制顶点 $d_{i}(i=0,1, \cdots, n)$, 在求解控制顶点时须知控制顶点 $d_{i}(i=0,1, \cdots, n)$ 对应的权因子 $\omega_{i}(i=0,1, \cdots, n)$, 在 求解权因子时, 通常为简化运算, 可以取 $\omega_{i}(i=0,1, \cdots, n)=1$, 也可以求型值点 $P_{i}=(i=0,1, \cdots$, $m)$ 对应的权值 $h_{i}(i=0,1, \cdots, m)$ 进行求解。

$$
\begin{gathered}
h_{i}=h\left(u_{i+3}\right)=\sum_{j=i}^{i+3} \omega_{j} N_{j, 3}\left(u_{i+3}\right) \\
u \in\left[u_{i+3}, u_{i+4}\right] \subset\left[u_{3}, u_{n+1}\right] \quad i=0,1, \cdots, m
\end{gathered}
$$

利用矩阵形式表示, 可得如下方程

$$
\left[\begin{array}{lll}
m_{11} & m_{12} & m_{13}
\end{array}\right]\left[\begin{array}{lll}
\omega_{i} & \omega_{i+1} & \omega_{i+2}
\end{array}\right]^{\mathrm{T}}=h_{i}
$$

式中, $i=0,1,2, \cdots, m$ 。

补充切失边界条件

$$
\begin{gathered}
3\left(w_{1}-w_{0}\right) / \Delta_{3}=h_{0}^{\prime} \\
3\left(w_{n}-w_{n-1}\right) / \Delta_{n}=h_{m}^{\prime}
\end{gathered}
$$

$h_{0}^{\prime}$ 和 $h_{m}^{\prime}$ 分别可以用数值微分得到

$$
h_{0}^{\prime}=\frac{h_{2}-h_{1}}{\Delta_{3}} h_{m}^{\prime}=\frac{h_{m}-h_{m-1}}{\Delta_{n}}
$$

由式(29) (31)可构成包含 $n+1$ 个方程的方程 组, 求解这些方程组, 就可得到各控制顶点对应的 权值 $\omega_{i}(i=0,1 \cdots n)$, 求解过程与求控制顶点 $d_{i}(i=0,1, \cdots, 3)$ 的过程相似。

\section{4 基于 NURBS 曲线的复杂曲面类零件分层算法 步骤}

本文采用基于 NURBS 曲线插值拟合的方法对 复杂曲面类零件分层, 具体步骤如下所述。
(1) 求取各切平面与 STL 模型上各三角面片的 交点, 构建所有交点的点云数据库, 并按切片层进 行分组排序。

(2) 对各层的交点数据进行处理, 以各层交点 数据作为 NURBS 曲线的型值点 $P_{i}=(i=0,1, \cdots, m)$, 并确定各型值点的权值 $h_{i}(i=0,1, \cdots, m)$, 取 NURBS 曲线的阶次 $k=3$, 按照式(15) (17) 确定节点矢量 $U=\left[u_{0}, u_{1}, \cdots, u_{n+k+1}\right]$, 其中, $n=m+2$ 。

(3) 根据式(28) (32)求解三次 NURBS 曲线的 权因子 $\omega_{i}(i=0,1, \cdots, n)$, 根据式(18) (27) 求解三次 NURBS 曲线的控制顶点 $d_{i}(i=0,1, \cdots, n)$ 。

(4) 根据式(10)可以求解出各切片层的截面轮 廓所对应的 NURBS 曲线方程。

(5) 绘制基于三次 NURBS 曲线拟合的各层层 面轮廓。

\section{3 基于 NURBS 曲线的复杂曲面类}

\section{零件分层算法实例}

\section{1 燃气轮机中压缸动叶片分层实例}

图 4 为某汽轮机有限公司生产的燃气轮机中压 缸动叶片, 叶片由叶根、叶型和叶冠构成, 叶根与 转子轮㪍配合安装在转子上, 叶型是气流流动的通 道, 其形状特点对汽轮机效率影响较大, 叶冠是叶 片与叶片之间配合的部位。

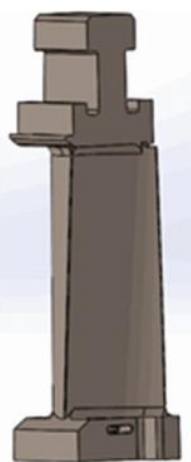

(a) 三维模型

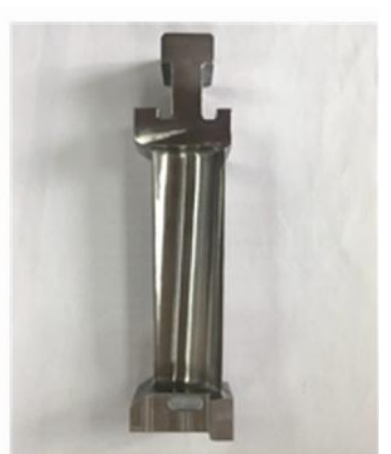

(b) 实物
图 4 燃气轮机中压缸动叶片

叶片叶型的型面特征参数最为复杂, 如图 5 所示, 叶片型线是由复杂的曲线线条构成, 在前 后缘点处, 曲率较大, 若对这一类零件进行分层 时，采用传统分层方法易造成分层面的轮廓线失 真, 因此, 采用基于 NURBS 曲线的复杂曲面类零 件分层截面生成算法对 STL 模型进行分层, NURBS 曲线的阶次取 3, 叶片的增材制造参数设 置如表 1 所示。 


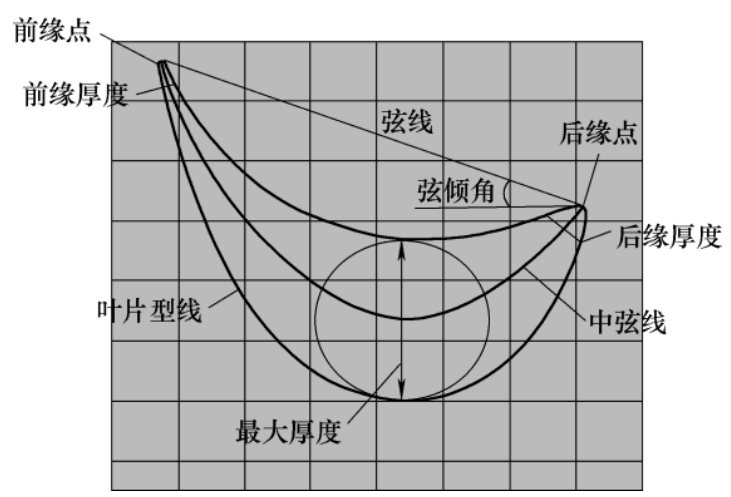

图 5 叶片型面特征参数
表 1 增材制造相关参数设置

\begin{tabular}{cccccc}
\hline $\begin{array}{c}\text { 打印 } \\
\text { 方式 }\end{array}$ & $\begin{array}{c}\text { 打印 } \\
\text { 材料 }\end{array}$ & $\begin{array}{c}\text { 材料直径 } \\
d / \mathrm{mm}\end{array}$ & $\begin{array}{c}\text { 填充密度 } \\
(\%)\end{array}$ & $\begin{array}{c}\text { 层高 } \\
\Delta Z / \mathrm{mm}\end{array}$ & 总层数 \\
\hline FDM & $\mathrm{ABS}$ & 1.75 & 100 & 0.2 & 742 \\
\hline
\end{tabular}

对燃气轮机中压缸动叶片进行分层，分层厚度 $0.2 \mathrm{~mm}$, 总层数 742 , 叶型部分的层面轮廓曲线较 复杂, 因此, 本文采用三次 NURBS 曲线对叶片叶 型部分的层面轮廓数据点进行插值拟合，叶片第 100 层、200 层、300 层、350 层、400 层、500 层的 层面轮廓插补拟合曲线如图 6 所示。采用基于三次

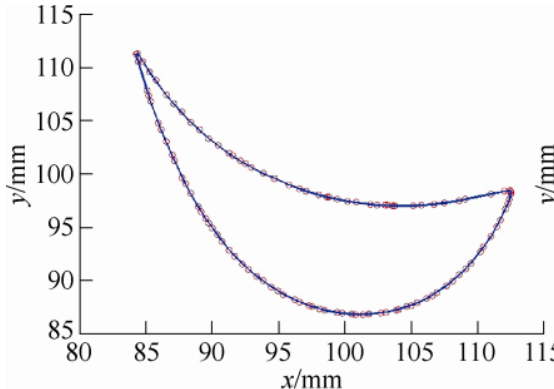

(a) 100 层

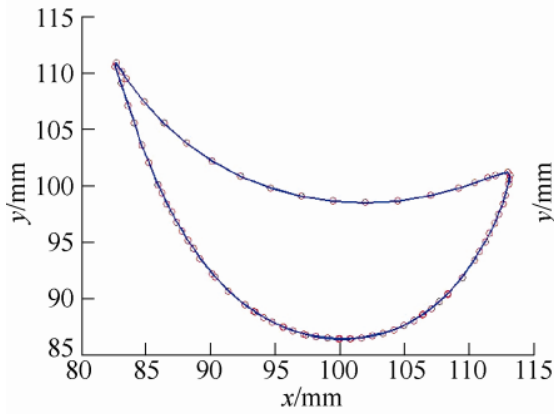

(d) 350 层

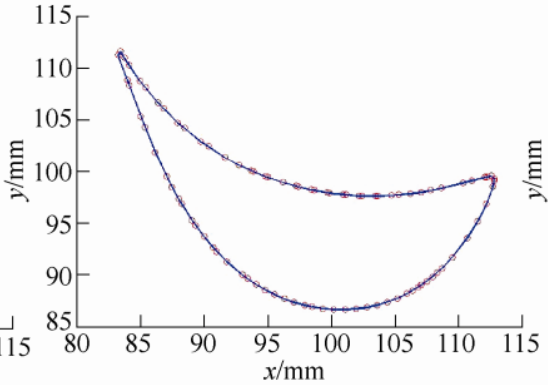

(b) 200 层

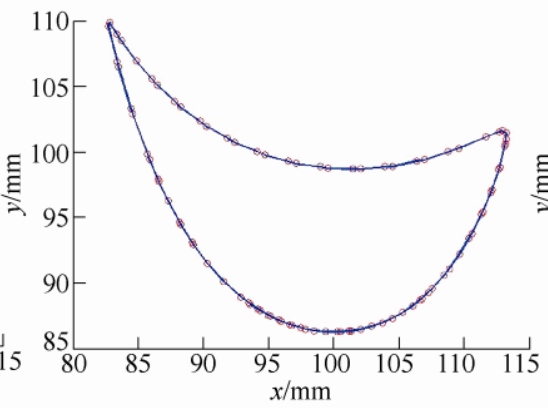

(e) 400 层

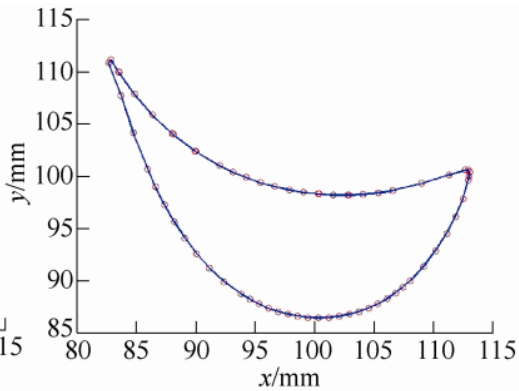

(c) 300 层

图 6 基于 NURBS 曲线的叶片分层截面插补拟合曲线

NURBS 曲线对叶片叶型部分各层的截面轮廓进行 插值拟合以及分层仿真, 其分层效果如图 7 所示。

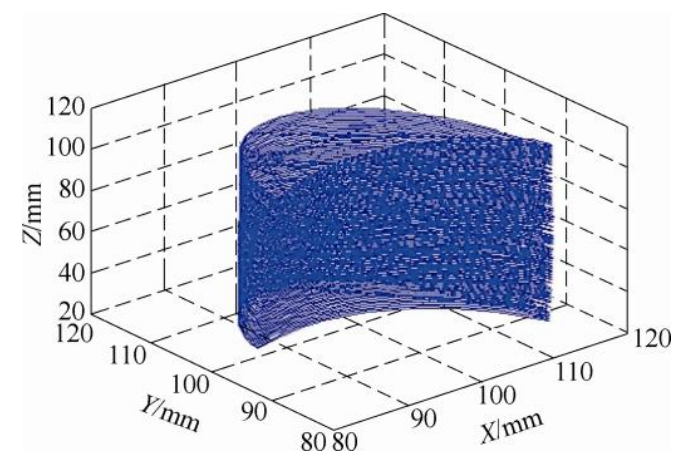

图 7 基于 NURBS 曲线的叶片叶型分层仿真图

\subsection{Ganesha 模型分层案例}

Ganesha (象头神) 模型是增材制造中常见的一 种三维模型, 如图 8 所示, 其曲面形状不规则, 对
Ganesha 模型进行分层，其各层的的轮廓曲线变化 速度快, 因此, 经常在一些分层算法中被使用。采 用基于 NURBS 曲线的复杂曲面类零件分层截面生 成算法进行分层, NURBS 曲线的阶次取 3, Ganesha 模型的增材制造参数设置如表 2 所示。

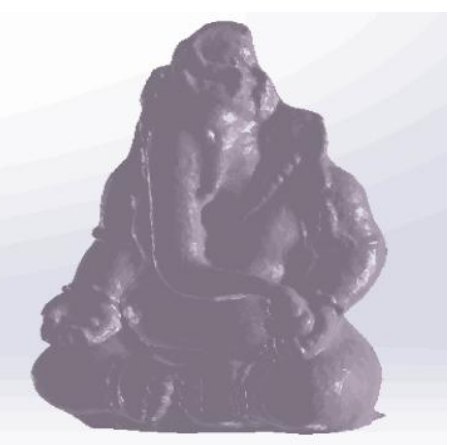

图 8 Ganesha 三维模型 
表2 增材制造相关参数设置

\begin{tabular}{cccccc}
\hline $\begin{array}{c}\text { 打印 } \\
\text { 方式 }\end{array}$ & $\begin{array}{c}\text { 打印 } \\
\text { 材料 }\end{array}$ & $\begin{array}{c}\text { 材料直径 } \\
d / \mathrm{mm}\end{array}$ & $\begin{array}{c}\text { 填充密 } \\
\text { 度 }(\%)\end{array}$ & $\begin{array}{c}\text { 层高 } \\
\Delta Z / \mathrm{mm}\end{array}$ & 总层数 \\
\hline $\mathrm{FDM}$ & $\mathrm{ABS}$ & 1.75 & 100 & 0.2 & 130 \\
\hline
\end{tabular}

对 Ganesha 模型进行分层, 分层厚度 $0.2 \mathrm{~mm}$, 总层数 130 , 采用三次 NURBS 曲线对
Ganesha 模型的各层面轮廓数据点进行插值拟 合，其第 20 层、40 层、60 层、80 层、100 层、 120 层的层面轮廓插补拟合曲线如图 9 所示。采 用三次 NURBS 曲线对 Ganesha 模型各层的截面 轮廓进行插值拟合以及分层仿真, 其分层效果如 图 10 所示。

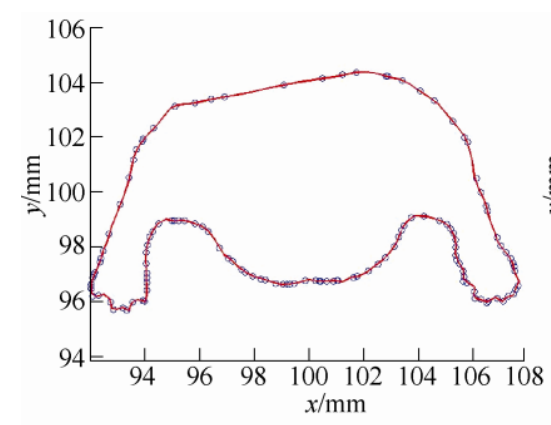

(a) 20 层

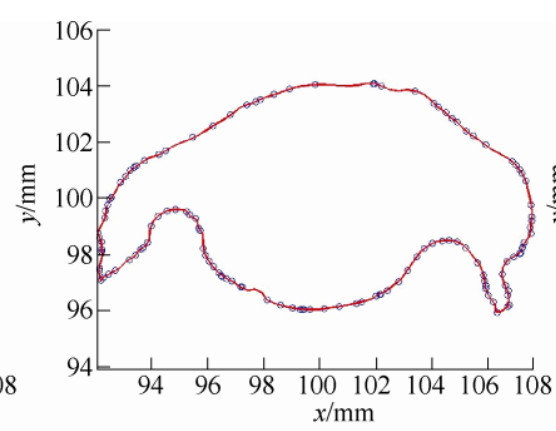

(b) 40 层

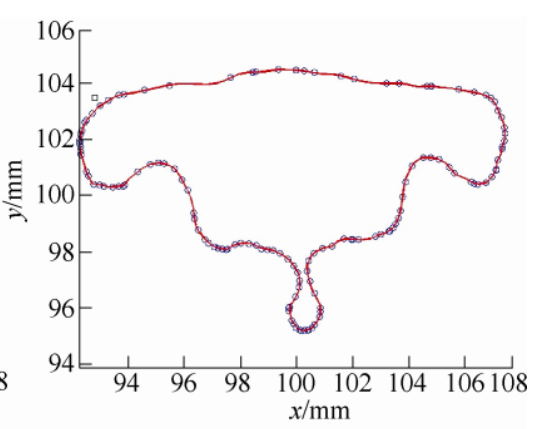

(c) 60 层

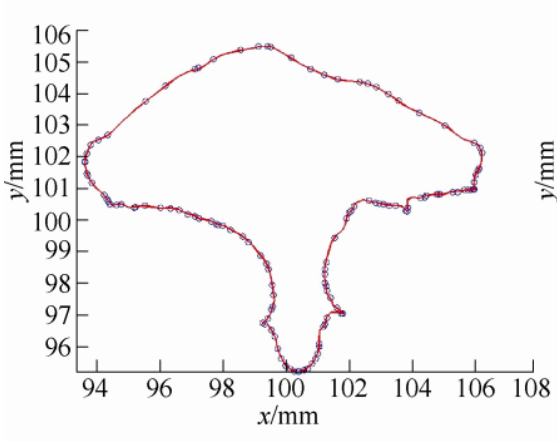

(d) 80 层

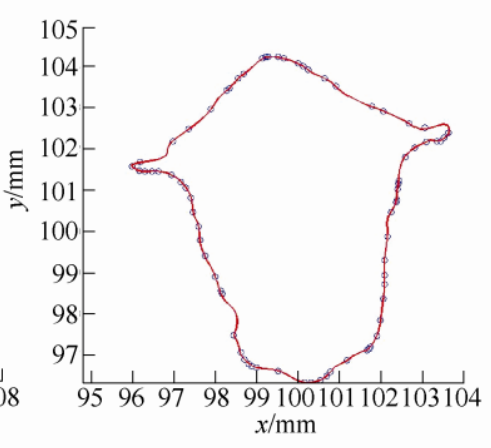

(e) 100 层

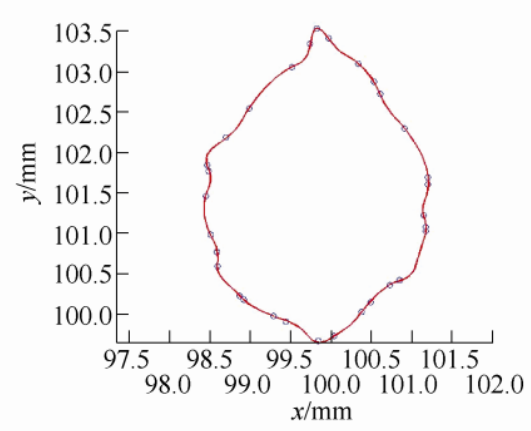

(f) 120 层

图 9 基于 NURBS 曲线的 Ganesha 模型分层截面插补拟合曲线

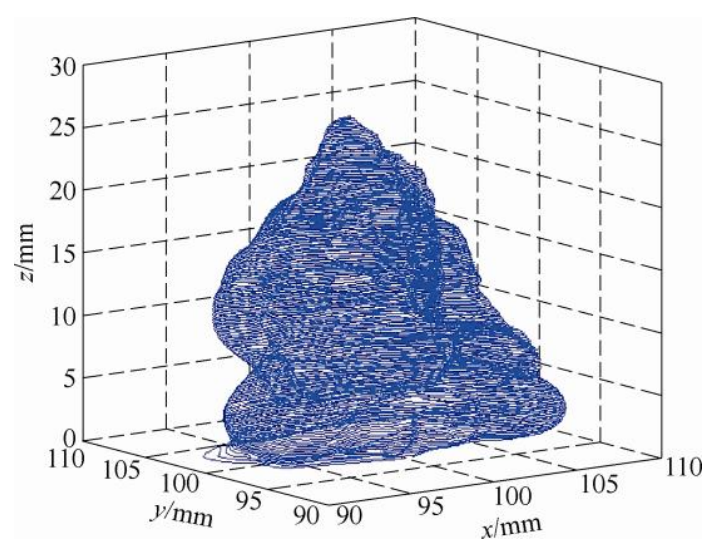

图 10 基于 NURBS 曲线的 Ganesha 模型分层仿真图

\section{3 基于 NURBS 曲线的层面轮廓误差分析}

假设增材制造零件在 $z=z_{i}$ 切片层上的一点 $M$, 在 $X O Y$ 平面的真实坐标值为 $\left(x_{M r}, y_{M r}\right)$, 假设采用 三次NURBS 曲线进行拟合的层面轮廓与 $M$ 点相对应 的点 $M^{\prime}$ 的坐标值为 $\left(x_{M p}, y_{M p}\right)$, 其中, $x_{M p}=x_{M r}$, 则基于 NURBS 曲线的分层算法的截面轮廓误差为

$$
e_{y}=\left|y_{M p}-y_{M r}\right|
$$

采用式(33)对基于三次 NURBS 曲线的燃气轮 机中压缸动叶片(图 4)分层截面轮廓进行层面轮廓 位置误差分析, 以第 500 层为例, 该层 $z$ 坐标值为 $100.300 \mathrm{~mm}$, 选取该层轮廓上 20 个数据点, 采用基 于 STL 模型传统算法的坐标值和基于三次 NURBS 曲线拟合的坐标值与这些数据点的真实坐标值的误 差如图 11 所示。

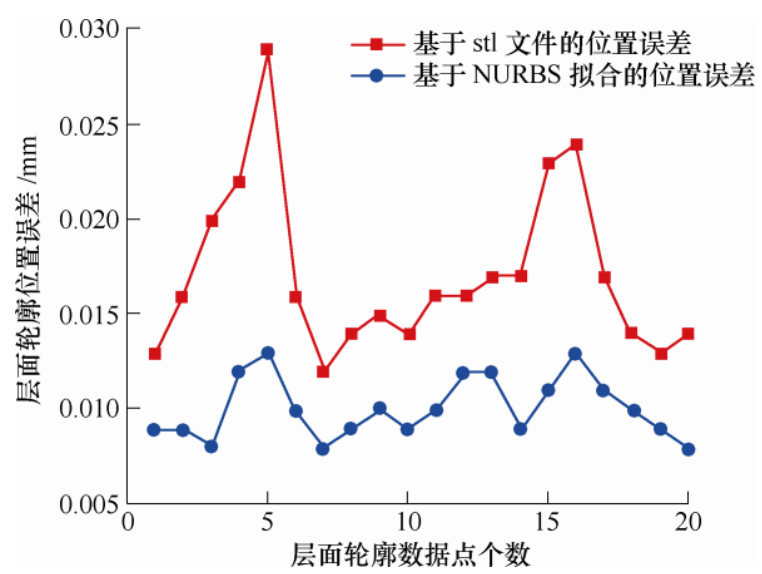

图 11 燃气轮机中压缸动叶片第 500 层 层面轮廓位置误差图 
此外, 对 Ganesha 模型(图 8)进行层面轮廓误差 分析, 以第 60 层层面轮廓为例, 该层 $z$ 坐标值为 $12.300 \mathrm{~mm}$, 选取该层轮廓上 20 个数据点, 采用基 于 STL 模型传统算法的坐标值和基于三次 NURBS 曲线拟合的坐标值与这些数据点的真实坐标值的误 差如图 12 所示。

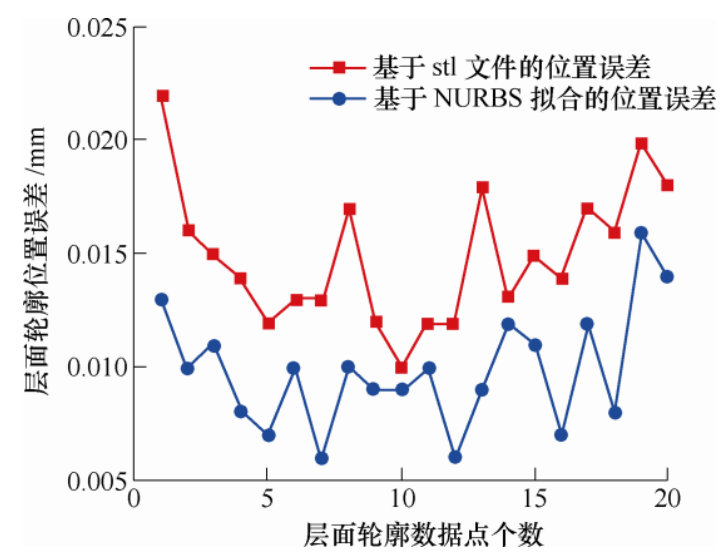

图 12 Ganesha 模型第 60 层截面轮廓位置误差图

由图 11 和图 12 可以看出, 采用基于三次 NURBS 曲线的层面轮廓位置误差比基于 STL 模型 传统算法的层面轮廓位置误差小，表明本文算法在 进行复杂曲面类零件层面轮廓生成方面比基于 STL 模型传统算法的计算精度更高。

需要说明的是, 层面轮廓数据点的位置误差 具有相对性, 因为层面轮廓的位置精度和 STL 模 型的三角面片个数和切片层数有关, 在切片层数 一定的情况下, 三角面片数量越多, 层面轮廓的 位置精度越高, 但所需的分层时间和打印时间更 长, 反之, 三角面片数量越少, 层面轮廓的位置 精度降低, 但分层效率和打印效率高。本文选用 的燃气轮机中压缸动叶片模型和 Ganesha 模型在 形成 STL 模型时, 采用中等数量的三角面片, 若 选用不同数量的三角面片, 层面轮廓的位置误差 也会有差异，但是在同等条件下，本文算法的位 置精度更高。

\section{4 打印试验}

在图 13 所示的三自由度机械臂熔融沉积式增 材制造样机上进行燃气轮机中压缸动叶片零件打印 试验, 增材制造材料选用 ABS, 打印喷头直径为 0.4 $\mathrm{mm}$, 分层厚度为 $0.2 \mathrm{~mm}$, 打印喷头在制造过程中 的移动速度为 $120 \mathrm{~mm} / \mathrm{s}$, 空行程的移动速度是 160 $\mathrm{mm} / \mathrm{s}$, 在对零件进行分层时, 采用本文提出的复杂 曲面类增材制造零件分层截面生成算法, 打印零件 如图 14b 所示。

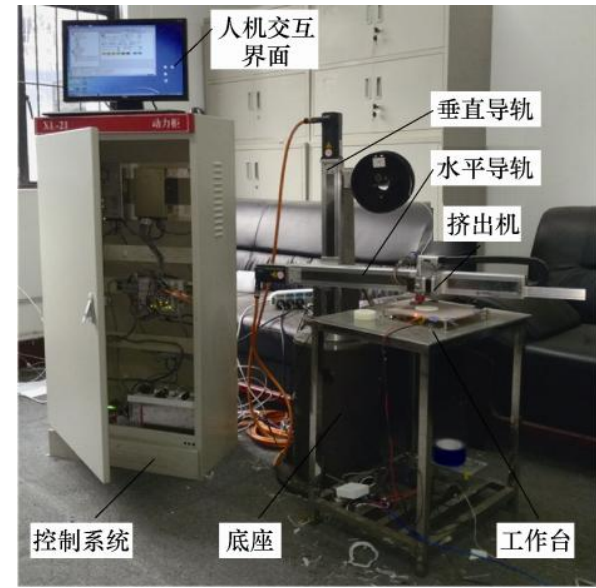

图 13 三自由度机械臂熔融沉积式增材制造样机

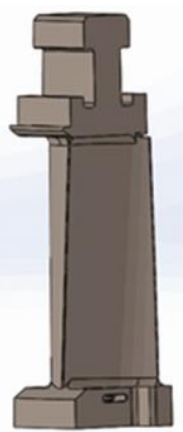

(a) 三维模型

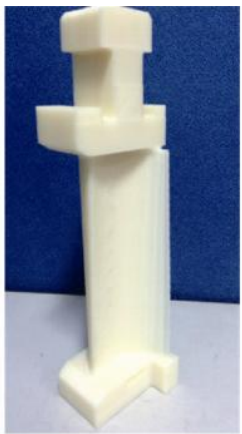

(b) 打印零件
图 14 叶片三维模型及打印零件

选取相同的打印参数和打印材料, 采用传统 STL 模型分层算法打印叶片实体零件, 采用三坐标 测量机对本文算法和传统算法打印的燃气轮机中压 缸动叶片叶型型面进行检测, 在叶片测量试验过程 中, 采集了距离叶根端面水平面距离为 $+32.5 \mathrm{~mm}$ 的 截面 B-B 和 $+65.00 \mathrm{~mm}$ 的截面 C-C 两个特定截面数 据, 如图 15 所示, 对两个特定截面进行型线轮廓度 偏差分析，采用本文算法的叶片型面型线轮廓度偏 差值如表 3 所示，采用传统算法的叶片型面型线轮 廓度偏差值如表 4 所示。

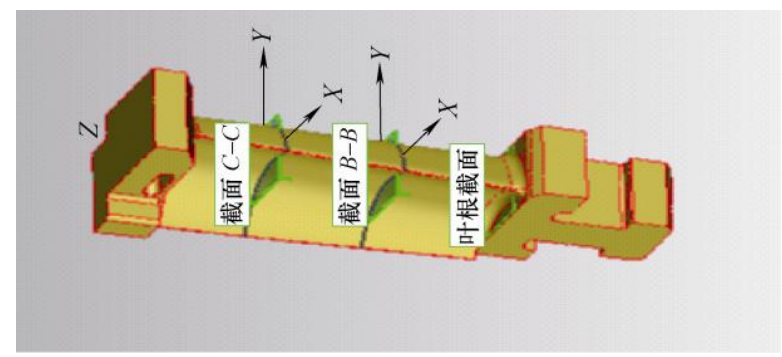

图 15 叶片检测截面

表 3 采用本文算法的叶片型面型线轮廓度偏差值

\begin{tabular}{cccc}
\hline 测量参数 & 最大偏差值 $/ \mathrm{mm}$ & 最小偏差值 $/ \mathrm{mm}$ & 标准偏差值 $/ \mathrm{mm}$ \\
\hline 截面 $B-B$ & 0.071 & -0.082 & 0.043 \\
截面 $C-C$ & 0.075 & -0.084 & 0.057 \\
\hline
\end{tabular}


表 4 采用传统 STL 模型分层算法叶片型面型线 轮廓度偏差值

\begin{tabular}{cccc}
\hline 测量参数 & 最大偏差值 $/ \mathrm{mm}$ & 最小偏差值 $/ \mathrm{mm}$ & 标准偏差值 $/ \mathrm{mm}$ \\
\hline 截面 $B-B$ & 0.101 & -0.098 & 0.062 \\
截面 $C-C$ & 0.097 & -0.105 & 0.073 \\
\hline
\end{tabular}

分别在叶片的 $B-B$ 截面和 $C-C$ 截面选取 10 个 位置点进行测量, 采用本文算法和传统算法打印零 件的轮廓度偏差绝对值如图 16 和图 17 所示。

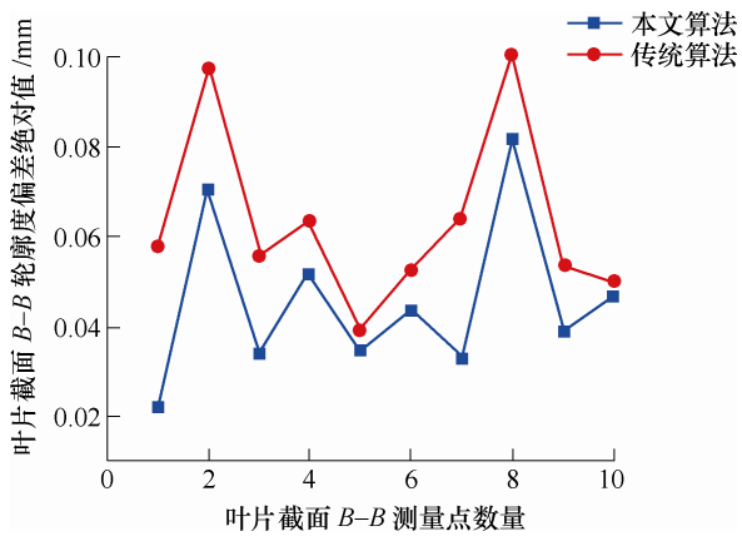

图 16 叶片截面 $B-B$ 轮廓度偏差绝对值

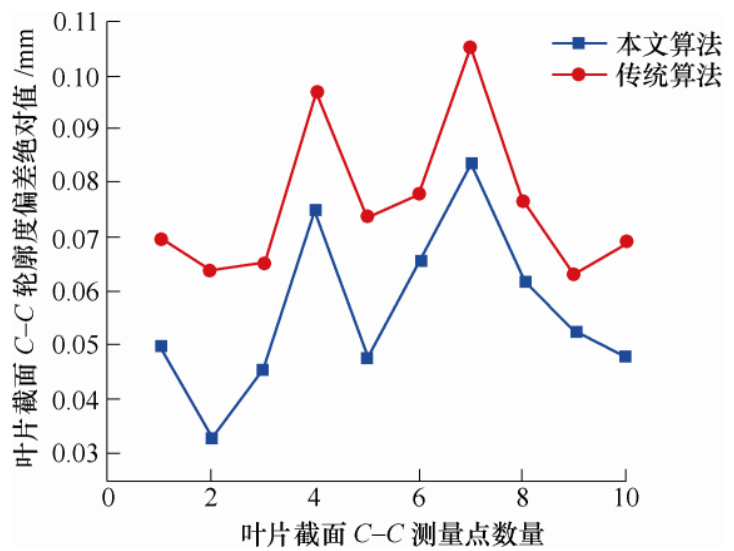

图 17 叶片截面 $C-C$ 轮廓度偏差绝对值

根据表 3、表 4、图 16 和图 17 可知, 采用本文 算法打印叶片的 $B-B$ 截面的型线轮廓度偏差值范围 为 $(-0.082 \sim 0.071 \mathrm{~mm})$, 标准偏差值为 $0.043 \mathrm{~mm}$; $C-C$ 截面的型线轮廓度偏差值范围为 $(-0.084 \sim$ $0.075 \mathrm{~mm}$ ), 标准偏差值为 $0.057 \mathrm{~mm}$; 采用传统算 法打印叶片的 $B-B$ 截面的型线轮廓度偏差值范围为 $(-0.098 \sim 0.101 \mathrm{~mm})$, 标准偏差值为 $0.062 \mathrm{~mm} ; C-C$ 截面的型线轮廓度偏差值范围为 $(-0.105 \sim 0.097$ $\mathrm{mm})$, 标准偏差值为 $0.073 \mathrm{~mm}$ 。已知叶片型线轮廓 度公差范围为 $\pm 0.1 \mathrm{~mm}$, 可见, 采用本文算法打印 的叶片两个关键截面的轮廓度偏差值符合要求, 采 用传统算法打印的叶片两个关键截面的轮廓度偏差 值存在不符合要求的位置点。不难看出, 采用本文 提出的基于 NURBS 曲线的复杂曲面类零件分层截
面生成算法打印的叶片零件在轮廓精度方面满足要 求，并且具有更高的精度，从而验证了本文算法的 可行性和准确性。

\section{5 结论}

(1) 针对复杂曲面类零件在分层时截面轮廓存 在失真问题，提出了一种基于 NURBS 曲线和 STL 模型的分层算法, 采用了 NURBS 曲线对截面轮廓 进行逼近拟合, 以切平面与 STL 模型三角面片的交 点作为 NURBS 曲线的型值点, 采用矩阵形式建立 了各切片层的截面轮廓所对应的 NURBS 曲线方 程，绘制了基于 NURBS 曲线的各层层面轮廓。

（2）采用基于 NURBS 曲线的复杂曲面类增材 制造零件分层截面生成算法对某燃气轮机中压缸动 叶片和 Ganesha 模型进行了分层试验仿真和层面轮 廓位置误差分析，结果表明：该算法比基于 STL 模 型的传统分层算法具有更高的位置精度。该算法可 用于解决采用传统方法对复杂曲面类零件分层造成 部分层面轮廓线失真的问题。

（3）采用熔融沉积式增材制造方式进行燃气轮 机中压缸动叶片零件打印试验, 对叶片叶型型面部 分进行精度检测, 结果表明: 采用本文提出的基于 NURBS 曲线的复杂曲面类零件分层截面生成算法 打印叶片的型面型线轮廓度偏差值能够满足要求, 而采用传统算法打印叶片的型面型线轮廓度偏差值 存在不符合要求的位置点。从而证明了本文提出的 基于 NURBS 曲线的复杂曲面类零件分层截面生成 算法具有较高的精度。

\section{参 考 文 献}

[1] 姜月娟, 卢秉恒, 方学伟, 等. 基于 3D 打印的网络化 集散制造模式研究[J]. 计算机集成制造系统, 2016, 22(6): 1424-1433.

JIANG Yuejuan, LU Bingheng, FANG Xuewei, et al. 3D printing based Internet collect manufacturing mode[J]. Computer Integrated Manufacturing System, 2016, 22(6): 1424-1433.

[2] 李涤尘, 贺健康, 田小永, 等. 增材制造: 实现宏微结 构一体化制造[J]. 机械工程学报, 2013, 49(6): 129-135. LI Dichen, HE Jiankang, TIAN Xiaoyong, et al. Additive manufacturing: Integrated fabrication of microstructures [J]. Journal of Mechanical Engineering, 2013, 49(6): $129-135$

[3] 刘利刚, 徐文鹏, 王伟明, 等. 3D 打印中的几何计算研 究进展[J]. 计算机学报, 2015，38(6)：1243-1267.

LIU Liugang, XU Wenpeng, WANG Weiming, et al. 
Survey on geometric computing in 3D printing[J]. Chinese Journal of Computers, 2015，38(6): 1243-1267.

[4] 石岩, 李云峰, 刘佳, 等. 高压油泵凸轮轴激光增材制 造梯度耐磨层研究 [J]. 机械工程学报, 2017, 53(6): 80-87.

SHI Yan, LI Yunfeng, LIU Jia, et al. Research of gradient wear-resisting coating produced by laser additive manufacturing on high-pressure pump camshaft[J]. Journal of Mechanical Engineering, 2017, 53(6): 80-87.

[5] 赵吉宾, 刘伟军. 快速成形技术中基于 stl 模型的分层 算法研究 $[J]$. 应用基础与工程科学学报, 2008, 16(2): 224-233.

ZHAO Jibin, LIU Weijun. Research on slicing algorithm based on STL model for rapid prototyping technology[J]. Journal of Basic Science and Engineering，2008， 16(2): 224-233.

[6] WANG W, CHAO H, TONG J, et al. Saliency-preserving slicing optimization for effective $3 \mathrm{D}$ printing[J]. Computer Graphics Forum, 2015， 34(6): 148-160.

[7] SUN S H, CHIANG H W, LEE M I. Adaptive direct slicing of a commercial CAD model for use in rapid prototyping[J]. International Journal of Advanced Manufacturing Technology, 2007, 34(7-8): 689-701.

[8] HOPE R L, JACOBS P A, ROTH R N. Rapid prototyping with sloping surfaces $[\mathrm{J}]$. Rapid Prototyping Journal, 1997, 3(1): 12-19.

[9] ROCK S J , WOZNY M J. Utilizing topological information to increase scan vector generation efficiency: Solid freeform fabrication symposium proceedings[R]. Austin: The University of Texas at Austin, 1991.

[10] 张必强, 邢滟, 阮雪榆. 面向网格简化的 STL 拓扑信 息快速重建算法 $[\mathrm{J}]$. 上海交通大学学报, 2004, 38(1): 39-42.

ZHANG Biqiang, XING Yuan, RUAN Xueyu. Fast generation of the topological information in STL for mesh simplification[J]. Journal of Shanghai Jiao Tong University, 2004, 38(1): 39-42.

[11] 谢存禧, 李仲阳, 成晓阳. STL 文件毗邻关系的建立与 切片算法研究[J]. 华南理工大学学报, 2000(3): 33-38. XIE Cunxi, LI Zhongyang, CHENG Xiaoyang. Constuction of the adjoining relationship of STL files and the study on slicing algorithm[J]. Journal of South China University of Technology, 2000(3): 33-38

[12] TATA K, FADEL G, BAGCHI A, et al. Efficient slicing for layered manufacturing[J]. Rapid Prototyping Journal, 1998, 4(4): 151-167.
[13] ZHANG Z, JOSHI S. An improved slicing algorithm with efficient contour construction using STL files[J]. International Journal of Advanced Manufacturing Technology, 2015, 80(5-8): 1347-1362.

[14] SABOURIN E, HOUSER S A, BOHN J H. Adaptive slicing using stepwise uniform refinement[J]. Rapid Prototyping Journal, 1996, 2(4): 20-26.

[15] SABOURIN E, HOUSER S A, BOHN J H. Accurate exterior, fast interior layered manufacturing[J]. Rapid Prototyping Journal, 1997, 3(2): 44-52.

[16] CORMIER D, UNNANON K, SANII E. Specifying non-uniform cusp heights as a potential aid for adaptive slicing[J]. Rapid Prototyping Journal, 2000, 6(3) : 204-211.

[17] PANDEY P M，REDDY N V，DHANDE S G. Real time adaptive slicing for fused deposition modelling $[\mathrm{J}]$. International Journal of Machine Tools \& Manufacture, 2003 , 43(PII S0890-6955(02)00164-51): 61-71.

[18] 刘斌, 黄树槐. 快速原型制造技术中实时切片算法的研 究与实现 [J]. 计算机辅助设计与图形学学报, 1997 , 9(6): 488-493.

LIU Bin, HUANG Shuhuai. Research and realization of real-time slicing algorithm in rapid prototyping \& manufacturing technology[J]. Journal of Computer- Aided Design \& Computer Graphics, 1997, 9(6): 488- 493.

[19] 施法中. 计算机辅助几何设计与非均匀有理 b 样条 [M]. 北京：高等教育出版社，2013。

SHI Fazhong. Computer-aided geometric design and non-uniform rational b-spline[M]. Beijing : Higher Education Press, 2013.

[20] PIEGL L, TILLER W. The NURBS book[M]. Germany Berlin: Springer, 1997.

[21] DIMITROV A , GU R , GOLPARVAR-FARD M. Non-uniform B-Spline surface fitting from unordered 3D point clouds for as-built modeling[J]. Computer-Aided Civil and Infrastructure Engineering, 2016, 31(7): 483-498.

[22] WU J, ZHOU H, TANG X, et al. Implementation of CL points preprocessing methodology with NURBS curve fitting technique for high-speed machining[J]. Computers \& Industrial Engineering，2015，81：58-64.

作者简介: 韩兴国, 男, 1981 年出生, 博士, 副教授。主要研究方向为 增材制造分层处理及路径规划方法。

E-mail: hanxingguo2004@163.com

殷国富(通信作者), 男, 1956 年出生, 博士, 教授, 博士研究生导师。 主要研究方向为 $\mathrm{CAD} / \mathrm{CAM}$ 、智能制造及装备。

E-mail: gfyin@scu.edu.cn 